\title{
Correction To: New obstructions to symplectic embeddings
}

\author{
R. Hind ${ }^{1}$ - E. Kerman ${ }^{2}$
}

Published online: 29 September 2018

(C) Springer-Verlag GmbH Germany, part of Springer Nature 2018

\section{Correction to: Invent. math. https://doi.org/10.1007/s00222-013-0471-2}

We correct here an error in the proof of Theorem 1.1 of our paper "New obstructions to symplectic embeddings," henceforth referred to as [6].

\section{The Error}

Theorem 1.1 depends on Theorem 2.36, an existence theorem for pseudoholomorphic curves. We first recall the statement of this existence theorem, rephrased in a more expansive form for convenience. In [6], the notation $\mathbb{C P}^{2}(R)$ is used for $\mathbb{C P}^{2}$ equipped with its standard symplectic structure multiplied by $R^{2}$ and $E(a, b)$ denotes the following symplectic ellipsoid in $\mathbb{C}^{2}$,

The original article can be found online at https://doi.org/10.1007/s00222-013-0471-2.

R. Hind

hind.1@nd.edu

E. Kerman

ekerman@math.uiuc.edu

1 Department of Mathematics, University of Notre Dame, Notre Dame, IN 46556, USA

2 Department of Mathematics, University of Illinois at Urbana-Champaign, Urbana, IL 61801, USA 


$$
E(a, b)=\left\{\frac{\left|z_{1}\right|^{2}}{a^{2}}+\frac{\left|z_{2}\right|^{2}}{b^{2}} \leq 1\right\}
$$

For $R>1$, any ellipsoid $E(a, b)$ with $a \leq b \leq 1$ can be identified with its image under its standard symplectic embedding into $\mathbb{C P}^{2}(R)$. With this, Theorem 2.36 of [6] is equivalent to the following.

Theorem 1 (Theorem 2.36 in [6]) Fix a natural number $d$ and an irrational number $S>\sqrt{3 d}$. Setting $E=E(1 / S, 1)$, let $\left(\mathbb{C P}^{2} \backslash E\right)_{-}^{\infty}$ be the negative symplectic completion of $\mathbb{C P}^{2}(R) \backslash E$. There exist an admissible almost complex structure $J$ on $\left(\mathbb{C P}^{2} \backslash E\right)_{-}^{\infty}$ and a regular finite energy J-holomorphic plane in $\left(\mathbb{C P}^{2} \backslash E\right)_{-}^{\infty}$ of degree $d$ whose negative end is asymptotic to the $(3 d-1)$-fold cover of the simple closed Reeb orbit on $E$ with period $\pi / S^{2}$.

Unfortunately, the proof presented in [6] is not correct for it also implies that the curves in the statement are embedded. The authors are very grateful to Dusa McDuff for pointing out that the existence of such embedded pseudo-holomorphic planes contradicts the results of Hutchings and Taubes from $[8,10,11]$. Indeed, as our curve satisfies the ECH partition conditions at its negative end, it turns out that the writhe inequality (see [8], section 3.3) must be an equality, which, in turn, would contradict the assertion that the curve is embedded. The error in [6] occurs in the proof of Lemma 2.53 where the possibility that the curves $G^{\epsilon}$ are multiply covered is not accounted for.

The proof of Theorem 1.5 in [6], which concerns embeddings into polydisks, does not rely on a degeneration near an exceptional divisor and so requires no changes.

\section{The Correction}

We will replace Theorem 1 with the following modified existence result. Let $\left\{g_{n}\right\}_{n \geq 1}$ be the odd index Fibonacci numbers.

Theorem 2 The conclusions of Theorem 1 hold if $d=g_{n+1}$ for some $n$.

This result can play the role of Theorem 2.36 in the original proof of Theorem 1.1 in [6] with only trivial adjustments. Indeed, the proof only requires the existence of the curves for a sequence of $d \rightarrow \infty$; see [6], section 3.4. We prove Theorem 2 in the next section. A compactness result, see [6], Proposition 3.4 , implies that it suffices to prove Theorem 2 for any symplectic embedding of $E$ into a $\mathbb{C P}^{2}(R)$.

Remark 1 A recent preprint of McDuff [15] (see Lemma 2.6) shows that Theorem 1 is in fact true as stated after all. As in the proof below, the argument in [15] utilizes the construction of cylinders in [7], but combines this with obstruction bundle gluing to produce planes for all $d$. 


\section{Proof of Theorem 2}

\subsection{Conventions}

It will be useful to have the following alternative notation for symplectic ellipsoids in $\mathbb{R}^{2 n}=\mathbb{C}^{n}$ :

$$
\mathcal{E}\left(a_{1}, \ldots, a_{n}\right)=\left\{\frac{\pi\left|z_{1}\right|^{2}}{a_{1}}+\cdots+\frac{\pi\left|z_{n}\right|^{2}}{a_{n}} \leq 1\right\}=E\left(\sqrt{\frac{a_{1}}{\pi}}, \ldots, \sqrt{\frac{a_{n}}{\pi}}\right) .
$$

The scaled ellipsoid $\mathcal{E}\left(c a_{1}, \ldots, c a_{n}\right)$ will be denoted simply by $c \mathcal{E}\left(a_{1}, \ldots, a_{n}\right)$. Similarly, we let $\mathcal{C P}(A)=\mathbb{C} P^{2}\left(\sqrt{\frac{A}{\pi}}\right)$ denote the projective plane scaled so that lines have area $A$. For an ellipsoid $\mathcal{E}(a, b)$ in $\mathbb{C}^{2}$, we will assume, unless otherwise stated, that $a<b$ and that $\frac{a}{b}$ is irrational. With this, there are exactly two simple closed Reeb orbits on the boundary of $\mathcal{E}(a, b)$. These orbits will be denoted by $\alpha$ and $\beta$. Their periods (actions) are $a$ and $b$, respectively. The $r^{\text {th }}$ iterate of any closed Reeb orbit $\gamma$ will be denoted by $\gamma^{(r)}$. The Conley-Zehnder index of the $k^{\text {th }}$ iterate of $\alpha$ is

$$
\mathrm{CZ}\left(\alpha^{(k)}\right)=2 k+2\left\lfloor\frac{k a}{b}\right\rfloor+1,
$$

and the Conley-Zehnder index of the $k^{\text {th }}$ iterate of $\beta$ is

$$
\mathrm{CZ}\left(\beta^{(k)}\right)=2 k+2\left\lfloor\frac{k b}{a}\right\rfloor+1
$$

\subsection{Overview}

The curve of Theorem 2 will be constructed by gluing together two types of curves. The centerpiece of the construction is the curve detected in [3] using Embedded Contact Homology. To the single negative end of this ECH curve, we will glue a holomorphic cylinder as detected in [7].

\subsection{Piece 1}

Fix a positive irrational number $S>3 g_{n+1}-1$ and set

$$
\mathcal{E}_{1}=\mathcal{E}(1, S)
$$


For $c_{1}>\frac{S g_{n}}{g_{n+2}}$ and $\epsilon>0$, let

$$
\mathcal{E}_{2}=c_{1} \mathcal{E}\left(1, \frac{g_{n+2}}{g_{n}}+\epsilon\right)
$$

The ellipsoid $\mathcal{E}_{1}$ is contained in $\operatorname{int}\left(\mathcal{E}_{2}\right)$, the interior of $\mathcal{E}_{2}$. Let $X_{1}^{2}$ be the standard symplectic completion of the compact symplectic cobordism $\mathcal{E}_{2} \backslash \operatorname{int}\left(\mathcal{E}_{1}\right)$ from $\partial \mathcal{E}_{1}$ to $\partial \mathcal{E}_{2}$. Let $\alpha_{i}$ be the faster simple closed Reeb orbit of $\partial \mathcal{E}_{i}$.

Proposition 1 For a generic admissible almost complex structure $J$ on $X_{1}^{2}$ and all $\epsilon>0$ sufficiently small, there is a regular J-holomorphic cylinder $u: \mathbb{R} \times S^{1} \rightarrow X_{1}^{2}$ of index zero whose negative end is asymptotic to $\alpha_{1}^{\left(3 g_{n+1}-1\right)}$ and whose positive end is asymptotic to $\alpha_{2}^{\left(g_{n+2}\right)}$.

Proposition 1 follows immediately from Theorem 2 of [7]. One only needs to verify that for all sufficiently small $\epsilon>0$, we have

$\mathrm{CZ}\left(\alpha_{1}^{\left(3 g_{n+1}-1\right)}\right)=2\left(3 g_{n+1}-1\right)+1=2 g_{n+2}+2\left(g_{n}-1\right)+1=\mathrm{CZ}\left(\alpha_{2}^{\left(g_{n+2}\right)}\right)$.

Here, we have used the recursion relation

$$
g_{n+2}=3 g_{n+1}-g_{n}
$$

that defines the odd index Fibonacci numbers.

\subsubsection{On the proof of Proposition 1}

For the sake of completeness, we outline a proof of Proposition 1 following [7].

In [17], Pardon establishes, among many other things, the invariance of Contact Homology with respect to the choice of contact forms. Given Eq. (3), and the fact that all closed Reeb orbits on irrational ellipsoids have odd ConleyZehnder indices, the following existence result is an immediate consequence of Pardon's work.

Proposition 2 [17] For any admissible almost complex structure $J$ on $X_{1}^{2}$, there exists a cylindrical J-holomorphic building $\mathbf{H}$ of index zero with one negative end asymptotic to $\alpha_{1}^{\left(3 g_{n+1}-1\right)}$ and one positive end asymptotic to $\alpha_{2}^{\left(g_{n+2}\right)}$.

Remark 2 A different proof of this existence result, ad hoc and concrete, is also given in [7]. 
The curves of the $J$-holomorphic building $\mathbf{H}$ map to either $X_{1}^{2}$ or to the symplectization of $\partial \mathcal{E}_{1}$ or $\partial \mathcal{E}_{2}$ which we will denote by $Y_{1}$ and $Y_{2}$. To prove Proposition 1, it suffices to show that for a generic choice of $J$, all the curves of $\mathbf{H}$ mapping to $Y_{1}$ or $Y_{2}$ must cover trivial cylinders and that the (now) unique curve of $\mathbf{H}$ mapping to $X_{1}^{2}$ is the desired regular cylinder.

First, we observe that since the building $\mathbf{H}$ is cylindrical and $X_{1}^{2}$ is an exact symplectic manifold, every curve of $\mathbf{H}$ has precisely one positive puncture. With this, one can establish the following alternative.

Lemma 1 If a curve $v$ of $\mathbf{H}$ maps to $Y_{1}$ or $Y_{2}$, then either the index of $v$ is positive, or $v$ has index zero and covers a trivial cylinder over a closed Reeb orbit.

This corresponds to Lemma 1 of [7]. The proof requires only the index formula for curves, simple inequalities involving the floor function, and Stokes Theorem.

Using Eq. (3), one can also obtain the following constraint on the indices of the other curves of $\mathbf{H}$.

Lemma 2 If $J$ is generic in the sense that every somewhere injective $J$ holomorphic curve of genus zero is regular, then each curve $u$ of $\mathbf{H}$ with image in $X_{1}^{2}$ has a nonnegative index.

This corresponds to Lemma 3 in [7]. The proof utilizes the ingredients above and the generic choice of $J$ to ensure that somewhere injective curves have nonnegative index. The condition that $S>3 g_{n+1}-1$ bounds the growth rate of the Conley-Zehnder index of covers of $\alpha_{1}$, and this in turn excludes multiple covers of negative index.

Lemmas 1 and 2 imply Proposition 1 up to the assertion that the cylinder $u$ is regular for generic $J$. In particular, since the sum of the indices of the curves of $\mathbf{H}$ is zero, the lemmas imply that curves mapping to $Y_{1}$ or $Y_{2}$ must all cover trivial cylinders, and hence there is a unique $J$-holomorphic curve $u$ of $\mathbf{H}$ which maps to $X_{1}^{2}$ and connects $\alpha_{1}^{\left(3 g_{n+1}-1\right)}$ to $\alpha_{2}^{\left(g_{n+2}\right)}$.

The fact that $u$ is regular for a generic choice of $J$ is established in Proposition 3 of [7] as follows. In Lemma 4 of [7], it is shown that for a generic choice of $J$, the curve $u$ covers a regular, somewhere injective $J$-holomorphic cylinder $v$ with index equal to zero. Corollary 3.17 of [18] then implies that $v$ is immersed. Since $u$ and $v$ are both cylinders, this implies that $u$ has no critical points. The regularity of $u$ then follows from Theorem 1 of [18].

Remark 3 There is a sequence $n_{k} \rightarrow \infty$ such that $\operatorname{gcd}\left(3 g_{n_{k}+1}-1, g_{n_{k}+2}\right)=1$. Indeed, these numbers are coprime provided $g_{n_{k}+2}$ is not divisible by 2 or 5 . For such $n_{k}$, the corresponding cylinder $u$ of Proposition 1 is somewhere injective. 


\subsection{Piece 2}

For any $c_{2}>1$ (and $\epsilon$ sufficiently small), it follows from [16] that there is a symplectic embedding $\Phi$ of the ellipsoid $\mathcal{E}_{2}$ into

$$
\mathcal{C P}^{2}\left(c_{2} c_{1} \frac{g_{n+2}}{g_{n+1}}\right) \text {. }
$$

Let $X_{2}^{3}$ be the symplectic completion of the symplectic cobordism $\mathcal{C} \mathcal{P}^{2}\left(c_{2} c_{1}\right.$ $\left.\frac{g_{n+2}}{g_{n+1}}\right) \backslash \Phi\left(\operatorname{int}\left(\mathcal{E}_{2}\right)\right)$. The following result is proved by Cristofaro-Gardiner and Hind in [3] as Proposition 5.

Proposition 3 Let $J$ be an admissible almost complex structure on $X_{2}^{3}$. If $\epsilon$ is sufficiently small and $c_{2}$ is sufficiently close to 1 , then there is a connected, embedded (and hence regular) $J$-holomorphic plane $v$ in $X_{2}^{3}$ of genus 0 , degree $g_{n+1}$ and with negative end which is asymptotic to $\alpha_{2}^{\left(g_{n+2}\right)}$.

Remark 4 Actually, [3] worked with the completion of a cobordism $\mathcal{E}_{3} \backslash$ $\Phi\left(\operatorname{int}\left(\mathcal{E}_{2}\right)\right)$ rather than $X_{2}^{3}$, where $\mathcal{E}_{3}=c_{2} c_{1} \mathcal{E}\left(\frac{g_{n+2}}{g_{n+1}}, \frac{g_{n+2}}{g_{n+1}}+\epsilon\right)$. However, identical reasoning applies to give Proposition 3; see also Remark 3.1.8(ii) in [4]. Alternatively, one could simply glue to the curves in [3] (which have positive ends asymptotic to simple covers of the slow orbit $\beta_{3}$ on $\mathcal{E}_{3}$ ) degree 1 planes in $\mathcal{C P}^{2}\left(c_{2} c_{1}\left(\frac{g_{n+2}}{g_{n+1}}+\epsilon\right)\right) \backslash \mathcal{E}_{3}$ with negative end asymptotic to the slow orbit. Such planes can be seen explicitly for the standard inclusion of $\mathcal{E}_{3}$.

The source of the curve from [3] is an isomorphism from the Embedded Contact Homology of $\partial \mathcal{E}_{3}$ to that of $\partial \mathcal{E}_{2}$ which is defined in terms of SeibergWitten theory. The orbit set $\beta_{3}^{g_{n+1}}$ represents a nontrivial ECH class, and the image of this class under the isomorphism is generated by the orbit set $\alpha_{2}^{g_{n+2}}$. This implies the existence of a broken J-holomorphic current whose cobordism component is the desired curve of Proposition 3. The proof uses the foundational work of Hutchings and Taubes from [8-14].

\subsection{Assembly and Completion}

Let $J_{1}$ be an admissible almost complex structure on $X_{1}^{2}$ which is generic in the sense of Proposition 1, and let $J_{2}$ be an admissible almost complex structure on $X_{2}^{3}$ which (eventually) agrees with $J_{1}$ on the negative end of $X_{2}^{3}$. Fix positive constants $\epsilon, c_{1}$ and $c_{2}$ so that the assertions of Propositions 1 and 3 hold, and let $u$ and $v$ be the regular pseudo-holomorphic curves promised by those propositions. Let $A=c_{2} c_{1} \frac{g_{n+2}}{g_{n+1}}$.

Denote by $\left(\mathcal{C P}^{2}(A) \backslash \operatorname{int}\left(\mathcal{E}_{1}\right)\right)_{-}^{\infty}$ the negative symplectic completion of $\mathcal{C P}^{2}(A) \backslash \operatorname{int}\left(\mathcal{E}_{1}\right)$. Since the curves $u$ and $v$ above are regular, we can apply 
the standard gluing procedure to glue $u$ to the negative puncture of $v$. The result is a regular pseudo-holomorphic plane in $\left(\mathcal{C P}^{2}(A) \backslash \operatorname{int}\left(\mathcal{E}_{1}\right)\right)_{-}^{\infty}$ of degree $g_{n+1}$ whose negative end is asymptotic to $\alpha_{1}^{\left(3 g_{n+1}\right)-1}$. This plane is $J$-holomorphic for some admissible $J$ which is stretched to a large finite length along the contact hypersurface corresponding to $\partial \mathcal{E}_{2}$. Rescaling in an obvious way, we can replace $\mathcal{E}_{1}$ by $E\left(\frac{1}{\sqrt{S}}, 1\right)$ and $\mathcal{C P}(A)$ by $\mathbb{C P}^{2}\left(\sqrt{\frac{A}{S}}\right)$ and the proof of Theorem 2 is complete.

\section{References}

1. Bourgeois, F., Eliashberg, Y., Hofer, H., Wysocki, K., Zehnder, E.: Compactness results in symplectic field theory. Geom. Topol. 7, 799-888 (2003)

2. Dragnev, D.: Fredholm theory and transversality for noncompact pseudoholomorphic maps in symplectizations. Commun. Pure Appl. Math. 57, 726-763 (2004)

3. Cristofaro-Gardiner, D., Hind, R.: Symplectic embeddings of products, to appear in Comment. Math. Helv. 93, 1-32 (2018)

4. Cristofaro-Gardiner, D., Hind, R., McDuff, D.: The ghost stairs stabilize to sharp symplectic embedding obstructions. J. Topol. 11, 309-378 (2018)

5. Eliashberg, Y., Givental, A., Hofer, H.: Introduction to Symplectic Field Theory, GAFA, Special Volume, pp. 560-673 (2000)

6. Hind, R., Kerman, E.: New obstructions to symplectic embeddings. Invent. Math. 196, 383-452 (2014)

7. Hind, R., Kerman, E.: J-holomorphic cylinders between ellipsoids in dimension four. arXiv: 1707.08635

8. Hutchings, M.: The embedded contact homology index revisited, New perspectives and challenges in symplectic field theory, CRM Proc. Lecture Notes 49. AMS, pp. 263-297 (2009)

9. Hutchings, M.: Lecture notes on embedded contact homology, contact and symplectic topology. Bolyai Soc. Math. Stud. 26, 389-484 (2014)

10. Hutchings, M., Taubes, C.: Gluing pseudoholomorphic curves along branched covered cylinders I. J. Symp. Geom. 5(1), 43-137 (2007)

11. Hutchings, M., Taubes, C.: Gluing pseudoholomorphic curves along branched covered cylinders II. J. Symp. Geom. 7(1), 29-133 (2009)

12. Hutchings, M., Taubes, C.: Proof of the Arnold chord conjecture in three dimensions I. MRL 18, 295-313 (2011)

13. Hutchings, M., Taubes, C.: Proof of the Arnold chord conjecture in three dimensions II. Geom. Topol. 17, 2601-2688 (2013)

14. Hutchings, M., Taubes, C.: The Weinstein conjecture for stable Hamiltonian structures. Geom. Topol. 13, 901-941 (2009)

15. McDuff, D.: On the stabilized symplectic embedding problem for ellipsoids. Eur. J. Math. 4, 356-371 (2018)

16. McDuff, D., Schlenk, F.: The embedding capacity of 4-dimensional symplectic ellipsoids. Ann. Math. 175, 1191-1282 (2012)

17. Pardon, J.: Contact homology and virtual fundamental cycles. arXiv: 1508.03873

18. Wendl, C.: Automatic transversality and orbifolds of punctured holomorphic curves in dimension four. Comment. Math. Helv. 85, 347-407 (2010) 